\title{
A machine learning approach to distinguish between knees without and with osteoarthritis using MRI-based radiomic features from tibial bone
}

\author{
Jukka Hirvasniemi $^{1}$ - Stefan Klein ${ }^{1}$ - Sita Bierma-Zeinstra ${ }^{2,3} \cdot$ Meike W. Vernooij $^{1,4} \cdot$ Dieuwke Schiphof $^{2}$. \\ Edwin H. G. Oei ${ }^{1}$ (D)
}

Received: 10 February 2021 / Revised: 1 March 2021 / Accepted: 25 March 2021 / Published online: 21 April 2021

(C) The Author(s) 2021

\begin{abstract}
Objectives Our aim was to assess the ability of semi-automatically extracted magnetic resonance imaging (MRI)-based radiomic features from tibial subchondral bone to distinguish between knees without and with osteoarthritis.

Methods The right knees of 665 females from the population-based Rotterdam Study scanned with 1.5T MRI were analyzed. A fast imaging employing steady-state acquisition sequence was used for the quantitative bone analyses. Tibial bone was segmented using a method that combines multi-atlas and appearance models. Radiomic features related to the shape and texture were calculated from six volumes of interests (VOIs) in the proximal tibia. Machine learning-based Elastic Net models with 10-fold cross-validation were used to distinguish between knees without and with MRI Osteoarthritis Knee Score (MOAKS)-based tibiofemoral osteoarthritis. Performance of the covariate (age and body mass index), image features, and combined covariate + image features models were assessed using the area under the receiver operating characteristic curve (ROC AUC).

Results Of 665 analyzed knees, 76 (11.4\%) had osteoarthritis. An ROC AUC of 0.68 (95\% confidence interval (CI): 0.60-0.75) was obtained using the covariate model. The image features model yielded an ROC AUC of 0.80 (CI: 0.73-0.87). The model that combined image features from all VOIs and covariates yielded an ROC AUC of 0.80 (CI: 0.73-0.87).

Conclusion Our results suggest that radiomic features are useful imaging biomarkers of subchondral bone for the diagnosis of osteoarthritis. An advantage of assessing bone on MRI instead of on radiographs is that other tissues can be assessed simultaneously.

Key Points

- Subchondral bone plays a role in the osteoarthritis disease processes.

- MRI radiomics is a potential method for quantifying changes in subchondral bone.

- Semi-automatically extracted radiomic features of tibia differ between subjects without and with osteoarthritis.
\end{abstract}

Keywords Magnetic resonance imaging $\cdot$ Radiomics $\cdot$ Knee osteoarthritis $\cdot$ Bone $\cdot$ Machine learning

Edwin H. G. Oei

e.oei@erasmusmc.nl

1 Department of Radiology \& Nuclear Medicine, Erasmus MC University Medical Center,

P.O. Box 2040CA 3000 Rotterdam, The Netherlands

2 Department of General Practice, Erasmus MC University Medical Center, Rotterdam, The Netherlands

3 Department of Orthopedics, Erasmus MC University Medical Center, Rotterdam, The Netherlands

4 Department of Epidemiology, Erasmus MC University Medical Center, Rotterdam, The Netherlands

\section{Abbreviations}

FIESTA Fast imaging employing steady-state acquisition

GLSZM Gray level size zone matrix

MOAKS MRI Osteoarthritis Knee Score

\section{Introduction}

Osteoarthritis is the most common joint disease affecting over 40 million people in Europe [1]. It reduces the quality of life of an individual and imposes a large economic burden on the society, since the direct and indirect costs can be as high as $2.5 \%$ of the gross domestic product of a nation [2,3]. Osteoarthritis affects all 
tissues in the joint, e.g., causing progressive degeneration of articular cartilage and changes in the subchondral bone density and structure $[4,5]$. Macroscopic alterations in the subchondral bone include osteophytes, bone sclerosis (stiffening), and cysts $[4,5]$. Advances in osteoarthritis diagnostics, prevention, and treatment will have a major impact on patients and society.

It has been recognized that subchondral bone plays a role in the pathogenesis of osteoarthritis [5-7] and subchondral bone has been suggested as a target for potential disease-modifying osteoarthritis drugs [8]. However, the exact role of subchondral bone in the development and progression of osteoarthritis is still unclear [5].

Magnetic resonance imaging (MRI) is considered the most comprehensive imaging modality for knee osteoarthritis assessment [9]. Semi-quantitative scoring systems have been developed to assess osteoarthritis-related structural deterioration of tissues on MRI [10]. Furthermore, many quantitative methods to assess articular cartilage and meniscus exist [11-13]. As subchondral bone is also involved in osteoarthritis disease processes, quantitative imaging biomarkers from bone might be helpful in the detection, prediction, and monitoring of the disease.

Radiomics is a field where a large number of quantitative image features (features related to intensity, geometric shape, and texture) are extracted from an image and correlated to biological markers and clinical outcomes. Radiomic methods have been successfully applied to different MRI data [14] but these methods have not yet been widely used for the assessment of knee osteoarthritis. Bone shape and texture variables have been previously extracted from knee MRI to assess osteoarthritic changes [15-18] and texture has been shown to correlate with the actual three-dimensional microstructure of subchondral bone [19]. However, previous texture analysis studies have manually defined regions of interests, analyzed limited number of slices, and used a limited number of texture variables.

As subchondral bone plays a role in the osteoarthritis disease processes, the aim of this study was to semi-automatically extract radiomic features from tibial subchondral bone using knee MRI data and to assess the ability of these radiomic features to distinguish between knees without and with osteoarthritis in a large population-based cohort.

\section{Subjects and methods}

\section{Study cohort}

The study data consisted of baseline data of 665 female participants from a sub-study (RS-III-1) of the Rotterdam Study, a population-based study in the Netherlands that investigates prevalence, incidence, and risk factors for various chronic disabling diseases [20-22]. Of the participants of the RS-III1 , the first 1116 females aged $45-60$ years were invited to join a sub-study investigating early signs of knee osteoarthritis [20, 21]. Of the 891 females who agreed to participate, 665 females with sufficient MR image quality and visual MRI grades available were included in the current study. The mean age and body mass index (BMI) of the subjects were 54.6 (standard deviation (SD): 3.7) years and 26.8 (SD: 4.6) kg/ $\mathrm{m}^{2}$, respectively. The Medical Ethics Committee of Erasmus University Medical Center approved the study and all subjects provided written informed consent.

\section{MRI acquisition}

All participants were scanned with a 1.5-T MRI scanner (Signa Excite 2, General Electric Healthcare) using an eightchannel cardiac coil that allowed imaging of both knees at once without repositioning the subject. The scanning protocol consisted of sagittal dual-echo fast spin echo (FSE) proton density-weighted, FSE T2-weighted with fat suppression, spoiled gradient echo with fat suppression, and fast imaging employing steady-state acquisition (FIESTA) sequences (Table 1).

\section{MRI assessment}

Two experienced readers scored the MRIs using the MRI Osteoarthritis Knee Score (MOAKS) [10]. The readers were extensively trained by an experienced musculoskeletal radiologist (E.O., > 15 years of experience with musculoskeletal MRI in clinical and research settings), as described previously [23].

Table 1 Parameters of the magnetic resonance imaging protocol

\begin{tabular}{lcccccc}
\hline Sequence & TR $(\mathrm{ms})$ & TE $(\mathrm{ms})$ & FOV $(\mathrm{mm})$ & Matrix & Flip angle (degrees) & Slice thickness/spacing (mm) \\
\hline Dual echo FSE PD & 2700 & 16 & $160 * 160$ & $512 * 512$ & 90 & $3.2 / 3.2$ \\
FSE T2-weighted FS & 4100 & 59 & $160 * 160$ & $512 * 512$ & 90 & $3.2 / 3.2$ \\
SGE FS & 27 & 6.3 & $160 * 160$ & $512 * 512$ & 30 & $3.2 / 1.6$ \\
FIESTA & 5.6 & 1.8 & $160 * 160$ & $512 * 512$ & 35 & $1.2 / 0.6$
\end{tabular}

FIESTA, fast imaging employing steady-state acquisition; $F S$, fat suppression; $F S E$, fast spin echo; $F O V$, field of view; $P D$, proton density; $S G E$, spoiled gradient echo; $T E$, time to echo; $T R$, repetition time 
We used a previously proposed definition for the identification of tibiofemoral osteoarthritis on MRI [24, 25]. Tibiofemoral osteoarthritis was defined as the presence of a definite osteophyte and full-thickness cartilage loss, or one of these features and two of the following features: (1) subchondral bone marrow lesion or cyst not associated with meniscal or ligamentous attachments, (2) meniscal maceration or degeneration (including a horizontal tear), or (3) partial thickness cartilage loss [24]. Grade 1 and 2 cartilage lesions were classified as partial thickness lesions and grade 3 lesions as full-thickness lesions. Grade 2 and 3 osteophytes were classified as definite osteophytes. Bone marrow lesions and cysts were present when scored as grade 1 or higher. Meniscusassociated features were present when maceration or degeneration had grade 1 or higher or a horizontal tear was present. We did not assess meniscal subluxation or bone attrition.

In addition to the tibiofemoral osteoarthritis, the ability of radiomic features to distinguish between knees without and with medial tibial cartilage damage, osteophytes, and bone marrow lesions were analyzed. A subject was included in the medial tibial cartilage damage group if she had any cartilage loss (grade 1 or higher for the size of any cartilage loss) in the medial anterior, central, or posterior tibia. Similarly, a subject was included in the medial tibial osteophytes group if she had osteophytes in the medial tibia (grade 1 or higher) and in the bone marrow lesion group if she had any bone marrow lesions (grade 1 or higher for the size of bone marrow lesions) in the medial anterior, central, or posterior part of tibia.

\section{Automatic segmentation of tibia}

The FIESTA scans were used in the quantitative analyses. Tibial bones from the right knees of the participants were segmented using an in-house automatic segmentation method that combines multi-atlas and appearance models [26, 27]. Twenty manually segmented tibias were used as atlases for the multi-atlas model and as training data for the appearance model. In the multi-atlas part, the atlas images were registered to the target image (i.e., the image to be segmented) using the Elastix software [28]. The registration was done by estimating an affine transformation followed by a non-rigid transformation. Mutual information was used as similarity measure for both transformations. The obtained registration parameters were used to deform the manual segmentations of the atlas images. The probability of a voxel in the target image to be part of the tibia segmentation or the background (i.e., non-tibia) was computed by averaging the deformed segmentations of all atlas images. The appearance model consisted of a random forest classifier that was trained on Gaussian scale space features in the training set. The feature vector (49 features) consisted of the intensity values and Gaussian-filtered version of the original images, first-order and second-order Gaussian derivatives in every axis direction, the gradient magnitude, the Laplacian, Gaussian Curvature, and the three eigenvalues of the Hessian of the Gaussian-filtered images. The classifier was then used to classify voxels on the target image to be either tibia or background. The final output of the segmentation method was obtained by combining the probabilities of the multi-atlas and appearance components. The segmentations were visually inspected and manually corrected if needed.

Altogether six three-dimensional volumes of interest (VOI) were automatically extracted from the medial and lateral compartments of the tibia (Fig. 1). Medial and lateral tibial spines were located by searching the highest and the second-highest coordinates along the vertical axis of the segmented sagittal slices. Because the positioning of the knee was similar among all scans, the medial and lateral compartments were identified using the tibial spines and outer borders of the tibia as landmarks. Medial and lateral subchondral bone VOIs were placed immediately below the cartilage-bone interface. The bottom coordinates for the abovementioned VOIs were defined as $10 \mathrm{~mm}$ below the cartilage-bone interface on the middle point of each compartment. Medial and lateral mid-part VOIs were placed under the subchondral bone VOIs. Medial and lateral trabecular bone VOIs were placed under the middle region VOIs. The height of the mid-part and trabecular bone VOIs was $10 \mathrm{~mm}$. It should be noted that despite referring the VOIs to as the subchondral bone, mid-part, and trabecular bone VOIs, different bone types are mixed in the VOIs $[29,30]$.

\section{Radiomics}

Radiomic features that are related to the shape and texture of the region were calculated from each VOI using the opensource Workflow for Optimal Radiomics Classification package (v. 3.0.0) in Python [31]. Seventeen shape features, 3

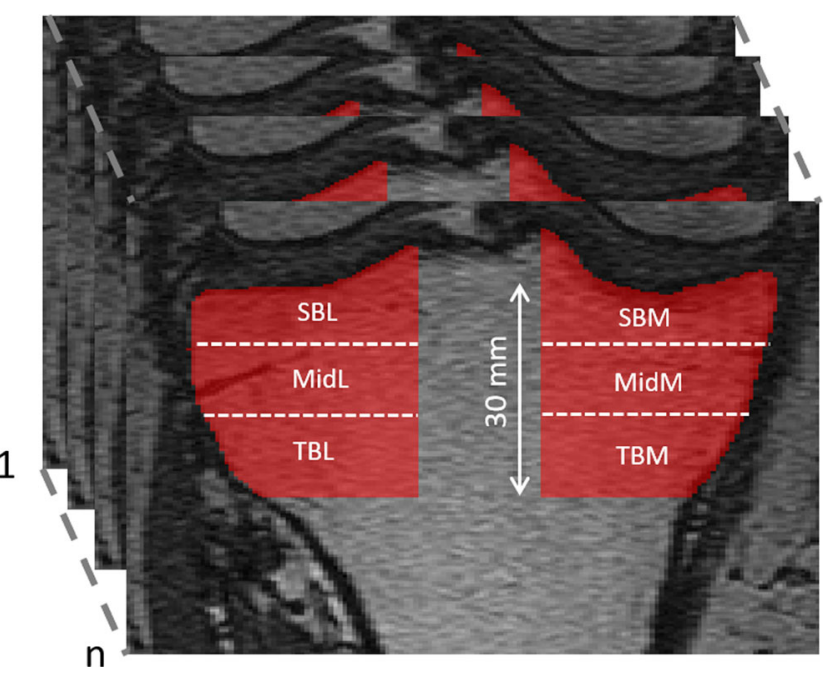

Fig. 1 3-D volumes of interest (VOIs) were extracted from medial (SBM) and lateral subchondral bone (SBL), mid-part of medial (MidM) and lateral (MidL) compartments, and medial (TBM) and lateral trabecular bone (TBL) 
orientation features, 12 histogram-based features, and 271 texture features were extracted (Supplementary Table 1). Texture features included local binary patterns, gray level cooccurrence matrix, gray level run length matrix, gray level size zone matrix (GLSZM), neighborhood gray tone difference matrix, and Gabor features. Features associated with shape were calculated only for the whole tibial volume.

\section{Statistical analyses}

To assess the accuracy of the automatic segmentation, the Dice similarity coefficient [32] was used to determine the overlap between five automatically and manually segmented tibias. These tibias were not included in the atlas set. The Dice coefficient ranges between 0 and 1 and higher value indicates better segmentation accuracy.

Machine learning was used for dimensionality reduction and to evaluate how well subjects without and with osteoarthritis can be distinguished using radiomics features from tibial bone. Three different models were used: (1) covariate model that included age and BMI that are two common risk factors for osteoarthritis [33], (2) image features model, and (3) combined covariate + image features model. Dimensionality reduction and classification were done using the Elastic Net regression, which is a regularized logistic regression method $[34,35]$. The Elastic Net linearly combines the L1 and L2 penalties of lasso and ridge regression methods. To optimize the ratio of the L1 and L2 penalties $(\alpha)$ and the strength of the penalty parameter $(\lambda)$ of the Elastic Net, 10-fold cross-validation with a grid search and 100 repetitions was performed. In the grid, the values of $\alpha$ varied from 0.1 to 1 with an increment of 0.05 and $\lambda$ from 0.001 to 0.15 with an increment of 0.009 . When $\alpha$ is close to 0 , the Elastic Net approaches ridge regression, while when $\alpha$ is 1 , lasso regression is performed. In cross-validation, the performance of the aforementioned three models to distinguish between subjects without and with osteoarthritis was assessed using the area under the receiver operating characteristic (ROC AUC) and precision-recall curves (PR AUC) along with $95 \%$ confidence intervals (CI) [36]. To assess the ability of radiomic features from medial tibial bone to distinguish individual osteoarthritis-related structural changes, analyses were repeated using medial tibial cartilage damage, osteophytes, and bone marrow lesions as an outcome variable. The Elastic Net experiments were done using the R software (version 3.5.2) with Caret [37], PRROC [38], glmnet [34], and precrec [39] packages.

\section{Results}

Of the 665 analyzed knees, 76 (11.4\%) had tibiofemoral osteoarthritis, 91 (13.7\%) had medial tibial cartilage damage, 85 $(12.8 \%)$ had a medial tibial osteophyte, and $70(10.5 \%)$ had a medial tibial bone marrow lesion. The mean Dice similarity coefficient for the uncorrected automatic segmentation of the tibia was 0.96 (SD: 0.02). Of the automatically segmented knees, 86 (12.9\%) were manually corrected.

When classifying knees without and with tibiofemoral osteoarthritis, ROC AUC and PR AUC of 0.80 (95\% CI: 0.73 0.87 ) and 0.46 (95\% CI: $0.33-0.58)$ were obtained with the model that combined image features from all VOIs and covariates (Table 2, Fig. 2). When each VOI was assessed separately, the medial subchondral bone VOI had the highest ROC AUC (Table 2). The model that combined image features from the medial subchondral bone VOI with covariates had an ROC AUC of 0.80 (95\% CI: 0.72-0.87) and a PR AUC of 0.46 (95\% CI: 0.33-0.58) (Supplementary Figure 1).

When classifying knees without and with medial tibial cartilage damage, the model that included image features from the medial subchondral bone VOI and covariates had the
Table 2 Area under the receiver operating characteristic curve (ROC AUC) and area under the precision-recall curve (PR AUC) values to distinguish between knees without and with osteoarthritis using models with only covariates (age and body mass index), only image features, and combined covariates and image features model for different volumes of interests (VOIs)

\begin{tabular}{|c|c|c|c|c|c|c|}
\hline \multirow[t]{2}{*}{ VOI } & \multicolumn{2}{|l|}{ Covariates } & \multicolumn{2}{|l|}{ Image features } & \multicolumn{2}{|c|}{ Covariates + image features } \\
\hline & ROC AUC & PR AUC & ROC AUC & PR AUC & ROC AUC & PR AUC \\
\hline All & $0.68(0.60-0.75)$ & $0.28(0.18-0.38)$ & $0.80(0.73-0.87)$ & $0.45(0.32-0.58)$ & $0.80(0.73-0.87)$ & $0.46(0.33-0.58)$ \\
\hline SBM & $0.68(0.60-0.75)$ & $0.28(0.18-0.38)$ & $0.79(0.71-0.86)$ & $0.46(0.33-0.58)$ & $0.80(0.72-0.87)$ & $0.46(0.33-0.58)$ \\
\hline MidM & $0.68(0.60-0.75)$ & $0.28(0.18-0.38)$ & $0.77(0.70-0.84)$ & $0.39(0.28-0.51)$ & $0.78(0.71-0.86)$ & $0.41(0.33-0.58)$ \\
\hline TBM & $0.68(0.60-0.75)$ & $0.28(0.18-0.38)$ & $0.74(0.67-0.81)$ & $0.33(0.22-0.44)$ & $0.76(0.68-0.83)$ & $0.35(0.24-0.46)$ \\
\hline SBL & $0.68(0.60-0.75)$ & $0.28(0.18-0.38)$ & $0.72(0.65-0.80)$ & $0.32(0.21-0.43)$ & $0.74(0.67-0.81)$ & $0.35(0.23-0.46)$ \\
\hline MidL & $0.68(0.60-0.75)$ & $0.28(0.18-0.38)$ & $0.73(0.66-0.80)$ & $0.33(0.22-0.44)$ & $0.75(0.68-0.82)$ & $0.35(0.24-0.47)$ \\
\hline TBL & $0.68(0.60-0.75)$ & $0.28(0.18-0.38)$ & $0.74(0.66-0.81)$ & $0.34(0.23-0.45)$ & $0.76(0.69-0.83)$ & $0.37(0.25-0.49)$ \\
\hline
\end{tabular}

All, all VOIs in the same model; SBM, medial subchondral bone VOI; MidM, medial mid-part VOI; TBM, medial trabecular bone VOI; SBL, lateral subchondral bone; MidL, lateral mid-part VOI; TBL, lateral trabecular bone VOI 


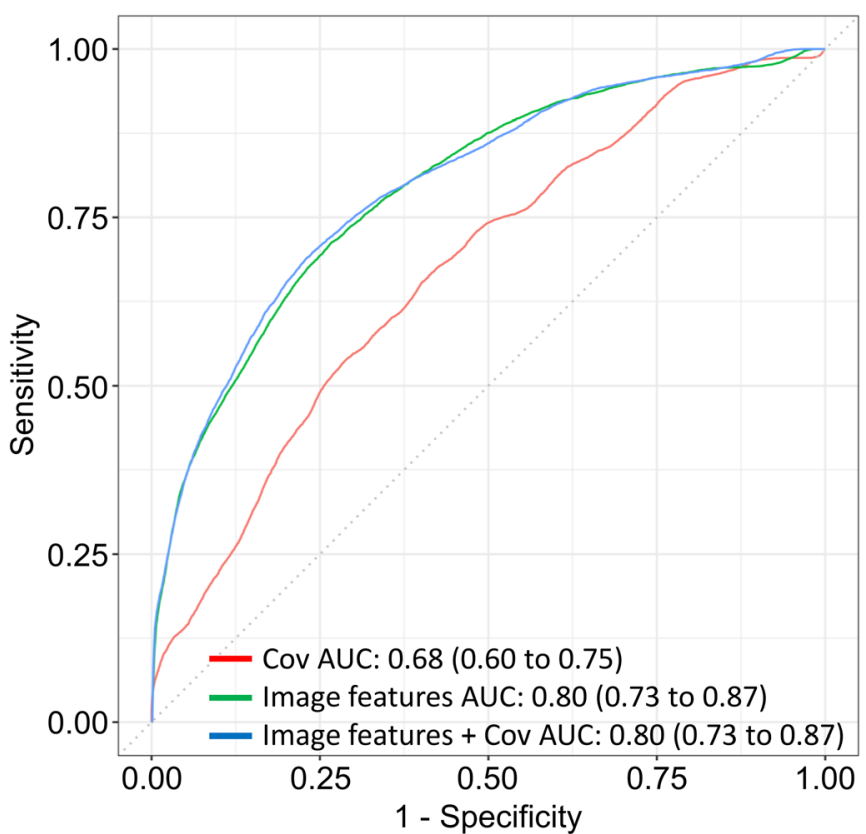

Fig. 2 Receiver operating characteristic and precision-recall curves and respective area under the curve (AUC) values to distinguish between knees without and with osteoarthritis using models with covariates (age

highest ROC AUC and PR AUC (0.66 (95\% CI: 0.59-0.73) and 0.30 (95\% CI: 0.21-0.39)) (Table 3, Supplementary Figure 2).

When classifying knees without and with medial tibial osteophytes, an ROC AUC of 0.80 (95\% CI: 0.73-0.86) and a PR AUC of 0.45 (95\% CI: 0.34-0.57) were obtained with the model that included image features from all medial side VOIs and covariates (Table 4, Supplementary Figure 3). When each VOI was assessed separately, medial mid-part VOI had the highest ROC AUC and PR AUC $(0.79$ (95\% CI: $0.73-0.89)$ and 0.44 (95\% CI: 0.33-0.56)) (Table 4).

When classifying knees without and with medial tibial bone marrow lesions, the model that included image features from the medial mid-part VOI and covariates had the highest ROC AUC and PR AUC (0.69 (95\% CI: 0.60-0.77) and 0.28 (95\% CI: 0.17-0.38)) (Table 5, Supplementary Figure 4).

Table 3 Area under the receiver operating characteristic curve (ROC AUC) and area under the precision-recall curve (PR AUC) values to distinguish between knees without and with medial tibial cartilage

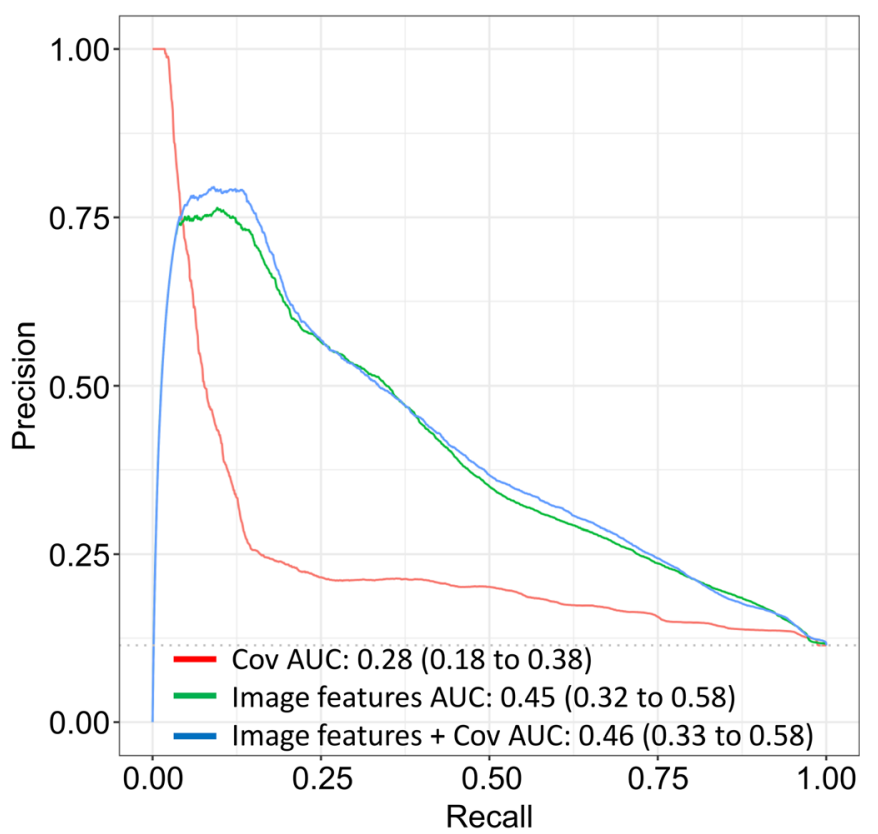

and body mass index), image features from all VOIs, and covariates and image features in the same model

The values for $\alpha$ and $\lambda$ hyperparameters and five variables with largest coefficients in the best performing Elastic Net models for classifying knees without and with tibiofemoral osteoarthritis, medial tibial cartilage damage, medial tibial osteophytes, and medial tibial bone marrow lesions are presented in Supplementary Table 2.

\section{Discussion}

In this study, we applied a semi-automatic segmentation method and extracted radiomic features from tibial bone using knee MRI data of a large population-based cohort. The highest ROC AUC and PR AUC for classifying knees without and with tibiofemoral osteoarthritis were obtained when all VOIs were combined in the same model. When each VOI was used separately in the model, image features from the medial

damage with the models using only covariates (age and body mass index), image features, and combined covariates and image features model for different volumes of interests (VOIs)

\begin{tabular}{|c|c|c|c|c|c|c|}
\hline \multirow[t]{2}{*}{ VOI } & \multicolumn{2}{|l|}{ Covariates } & \multicolumn{2}{|l|}{ Image features } & \multicolumn{2}{|c|}{ Covariates + image features } \\
\hline & ROC AUC & PR AUC & ROC AUC & PR AUC & ROC AUC & PR AUC \\
\hline All medial & $0.60(0.52-0.69)$ & $0.21(0.15-0.27)$ & $0.62(0.54-0.69)$ & $0.27(0.18-0.36)$ & $0.62(0.54-0.70)$ & $0.27(0.18-0.36)$ \\
\hline SBM & $0.60(0.52-0.69)$ & $0.21(0.15-0.27)$ & $0.66(0.59-0.73)$ & $0.29(0.20-0.38)$ & $0.66(0.59-0.73)$ & $0.30(0.21-0.39)$ \\
\hline MidM & $0.60(0.52-0.69)$ & $0.21(0.15-0.27)$ & $0.58(0.50-0.66)$ & $0.22(0.15-0.29)$ & $0.59(0.51-0.67)$ & $0.22(0.15-0.30)$ \\
\hline TBM & $0.60(0.52-0.69)$ & $0.21(0.15-0.27)$ & $0.55(0.47-0.63)$ & $0.20(0.13-0.26)$ & $0.55(0.48-0.63)$ & $0.20(0.14-0.26)$ \\
\hline
\end{tabular}

All medial, all medial VOIs in the same model; SBM, medial subchondral bone VOI; MidM, medial mid-part VOI; TBM, medial trabecular bone VOI 
Table 4 Area under the receiver operating characteristic curve (ROC AUC) and area under the precision-recall curve (PR AUC) values to distinguish between knees without and with medial tibial osteophytes with the models using only covariates (age and body mass index), image features, and combined covariates and image features model for different volumes of interests (VOIs)

\begin{tabular}{|c|c|c|c|c|c|c|}
\hline \multirow[t]{2}{*}{ VOI } & \multicolumn{2}{|l|}{ Covariates } & \multicolumn{2}{|l|}{ Image features } & \multicolumn{2}{|c|}{ Covariates + image features } \\
\hline & ROC AUC & PR AUC & ROC AUC & PR AUC & ROC AUC & PR AUC \\
\hline All medial & $0.64(0.56-0.72)$ & $0.28(0.18-0.37)$ & $0.80(0.73-0.86)$ & $0.45(0.34-0.57)$ & $0.80(0.73-0.86)$ & $0.45(0.34-0.57)$ \\
\hline SBM & $0.64(0.56-0.72)$ & $0.28(0.18-0.37)$ & $0.76(0.69-0.83)$ & $0.41(0.29-0.53)$ & $0.77(0.69-0.84)$ & $0.43(0.31-0.55)$ \\
\hline MidM & $0.64(0.56-0.72)$ & $0.28(0.18-0.37)$ & $0.79(0.73-0.86)$ & $0.45(0.33-0.56)$ & $0.79(0.73-0.89)$ & $0.44(0.33-0.56)$ \\
\hline TBM & $0.64(0.56-0.72)$ & $0.28(0.18-0.37)$ & $0.73(0.66-0.80)$ & $0.34(0.24-0.44)$ & $0.73(0.65-0.80)$ & $0.37(0.26-0.48)$ \\
\hline
\end{tabular}

All medial, all medial VOIs in the same model; SBM, medial subchondral bone VOI; MidM, medial mid-part VOI; TBM, medial trabecular bone VOI

subchondral bone and mid-part VOIs had the highest ROC AUC and PR AUC. This can be explained by the fact that the medial side of the knee is more commonly affected in osteoarthritis than the lateral compartment [40, 41].

For comprehensiveness, we also tested if the radiomic features are able to distinguish between subjects without and with medial tibial cartilage damage, osteophytes, and bone marrow lesions. Image features from the medial subchondral bone VOI had the highest ROC AUC and PR AUC to distinguish between knees without and with medial tibial cartilage damage indicating an interplay between cartilage and subchondral bone. Image features from the medial mid-part VOI had the highest ROC AUC and PR AUC to distinguish between knees without and with medial tibial bone marrow lesions. The model that combined all medial side VOIs in the same model had the highest ROC AUC and PR AUC to distinguish between knees without and with medial tibial osteophytes. These findings suggest that in addition to the subchondral bone area closest to the cartilage-bone interface, the characteristics of deeper bone areas are also altered in osteoarthritis.

The number of studies using radiomic approach for assessment of bone and osteoarthritis is limited, but texture variables have been previously extracted from knee MRI to assess osteoarthritic changes in bone $[16,17,19]$. One study predicted progression of knee osteoarthritis over 36 months (defined using joint space narrowing on plain radiographs) using bone texture on MRI and reported ROC AUCs between 0.58 and 0.68 depending on the model [15]. Radiography-based bone texture has been used to predict progression or development of osteoarthritis (ROC AUCs between 0.65 and 0.85) [42-44] and to distinguish between knees without and with osteoarthritis (ROC AUCs between 0.81 and 0.85) [45, 46]. The advantage of assessing bone on MRI is that other tissues involved in osteoarthritis can be assessed simultaneously. Furthermore, a two-dimensional plain radiograph is a projection of a three-dimensional structure, whereas MRI can produce three-dimensional data from the target structure.

When looking at the variables related to the geometrical shape of the bone, the compactness variable was included in all best performing models for classifying each of the used outcomes. The compactness variable describes how compact the bone shape on the sagittal slice is compared to a circle [14, 47]. By inspecting the direction of the coefficients in the final Elastic Net regression models, our results indicate that lower compactness is associated with tibiofemoral osteoarthritis.

Although many texture features contributed to the final models, Gabor and GLSZM were present in many models. Gabor filters respond to the edges (changes in the voxel intensities) in an image and give information about the texture in certain directions $[14,48,49]$. GLSZM quantifies gray level
Table 5 Area under the receiver operating characteristic curve (ROC AUC) and area under the precision-recall curve (PR AUC) values to distinguish between knees without and with medial tibial bone marrow lesions with the models using only covariates (age and body mass index), image features, and combined covariates and image features model for different volumes of interests (VOIs)

\begin{tabular}{|c|c|c|c|c|c|c|}
\hline \multirow[t]{2}{*}{ VOI } & \multicolumn{2}{|l|}{ Covariates } & \multicolumn{2}{|l|}{ Image features } & \multicolumn{2}{|c|}{ Covariates + image features } \\
\hline & ROC AUC & PR AUC & ROC AUC & PR AUC & ROC AUC & PR AUC \\
\hline All medial & $0.63(0.54-0.72)$ & $0.24(0.14-0.34)$ & $0.68(0.59-0.76)$ & $0.23(0.15-0.32)$ & $0.68(0.60-0.77)$ & $0.25(0.16-0.35)$ \\
\hline SBM & $0.63(0.54-0.72)$ & $0.24(0.14-0.34)$ & $0.65(0.57-0.73)$ & $0.21(0.13-0.29)$ & $0.67(0.59-0.75)$ & $0.25(0.15-0.34)$ \\
\hline MidM & $0.63(0.54-0.72)$ & $0.24(0.14-0.34)$ & $0.67(0.59-0.76)$ & $0.25(0.15-0.34)$ & $0.69(0.60-0.77)$ & $0.28(0.17-0.38)$ \\
\hline TBM & $0.63(0.54-0.72)$ & $0.24(0.14-0.34)$ & $0.57(0.48-0.66)$ & $0.19(0.11-0.27)$ & $0.58(0.49-0.68)$ & $0.21(0.12-0.30)$ \\
\hline
\end{tabular}

All medial, all medial VOIs in the same model; SBM, medial subchondral bone VOI; MidM, medial mid-part VOI; TBM, medial trabecular bone VOI 
zones (i.e., the number of connected voxels that share the same gray level intensity) in an image [14]. The direction of the coefficients in the Elastic Net models indicate that more homogeneous bone texture is associated with osteoarthritis. One explanation for this is that the subjects with osteoarthritis may have thickened subchondral bone which can lead to more homogeneous texture.

One strength of this study was the application of an automatic multi-atlas and appearance model-based algorithm for the segmentation of tibias. Although different MRI sequences and data were used, the performance of our segmentation method was comparable to the previously reported method for the segmentation of tibial bone [50]. It should be mentioned that our method was not fully automatic because $13 \%$ of the segmentations needed manual adjustment.

This study has limitations that need to be addressed. First, the radiomic features were extracted only from the tibia. In future studies, features could be extracted from other bones as well. Second, the link between biological processes and individual radiomic features is not always clear. However, MRI-based bone texture has been shown to correlate with the actual three-dimensional microstructure of subchondral bone [19]. Third, only basic risk factors of osteoarthritis were used as covariates in the models. In future studies, models with more risk factors should be used and the additional value of radiomic features on the diagnosis and prognosis of osteoarthritis should be studied.

In conclusion, our results show that radiomic features of tibial bone are different between knees without and with osteoarthritis and could be used as quantitative imaging biomarkers in future studies. As MRI enables assessment of multiple tissues in a joint, extraction of quantitative imaging biomarkers from bone would be beneficial in order to get a comprehensive view of the tissue changes associated with osteoarthritis.

Supplementary Information The online version contains supplementary material available at https://doi.org/10.1007/s00330-021-07951-5.

Acknowledgements The authors would like to thank all the participants and staff of the Rotterdam Study.

Funding This project has received funding from the European Union's Horizon 2020 research and innovation programme under the Marie Sklodowska Curie grant agreement no 707404. The Rotterdam Study is financially supported by the Netherlands Organisation of Scientific Research NWO Investments (no. 175.010.2005.011, 911-03-012), the Research Institute for Diseases in the Elderly (014-93-015; RIDE2), the Netherlands Genomics Initiative (NGI)/Netherlands Organisation for Scientific Research (NWO) (project no. 050-060-810), Netherlands Consortium of Healthy Aging, and the Erasmus Medical Center and
Erasmus University Rotterdam. The funding sources had no role in the study design, data collection or analysis, interpretation of data, writing of the manuscript, or in the decision to submit the manuscript for publication.

\section{Declarations}

Guarantor The scientific guarantor of this publication is Edwin Oei.

Conflict of interest The authors of this manuscript declare no relationships with any companies whose products or services may be related to the subject matter of the article.

Statistics and biometry JH and SK have significant statistical expertise related to this study.

Informed consent Written informed consent was obtained from all subjects in this study.

Ethical approval The Medical Ethics Committee of Erasmus University Medical Center approved the study.

Study subjects or cohorts overlap The study subjects and the cohort have been previously reported for example in [20-22].

\author{
Methodology \\ - retrospective \\ - cross-sectional study \\ - performed at one institution
}

Open Access This article is licensed under a Creative Commons Attribution 4.0 International License, which permits use, sharing, adaptation, distribution and reproduction in any medium or format, as long as you give appropriate credit to the original author(s) and the source, provide a link to the Creative Commons licence, and indicate if changes were made. The images or other third party material in this article are included in the article's Creative Commons licence, unless indicated otherwise in a credit line to the material. If material is not included in the article's Creative Commons licence and your intended use is not permitted by statutory regulation or exceeds the permitted use, you will need to obtain permission directly from the copyright holder. To view a copy of this licence, visit http://creativecommons.org/licenses/by/4.0/.

\section{References}

1. Conaghan PG, Kloppenburg M, Schett G, Bijlsma JWJ (2014) Osteoarthritis research priorities: a report from a EULAR ad hoc expert committee. Ann Rheum Dis 73:1442-1445

2. Salmon JH, Rat AC, Sellam J et al (2016) Economic impact of lower-limb osteoarthritis worldwide: a systematic review of costof-illness studies. Osteoarthritis Cartilage 24:1500-1508

3. Hunter DJ, Schofield D, Callander E (2014) The individual and socioeconomic impact of osteoarthritis. Nat Rev Rheumatol 10:437-441

4. Buckwalter JA, Mankin HJ (1998) Articular cartilage: degeneration and osteoarthritis, repair, regeneration, and transplantation. Instr Course Lect 47:487-504

5. Weinans H, Siebelt M, Agricola R, Botter SM, Piscaer TM, Waarsing JH (2012) Pathophysiology of peri-articular bone changes in osteoarthritis. Bone 51:190-196 
6. Radin EL, Rose RM (1986) Role of subchondral bone in the initiation and progression of cartilage damage. Clin Orthop Relat Res 34-40

7. Mansell JP, Collins C, Bailey AJ (2007) Bone, not cartilage, should be the major focus in osteoarthritis. Nat Clin Pract Rheumatol 3:306-307

8. Kwan Tat S, Lajeunesse D, Pelletier JP, Martel-Pelletier J (2010) Targeting subchondral bone for treating osteoarthritis: what is the evidence? Best Pract Res Clin Rheumatol 24:51-70

9. Roemer FW, Crema MD, Trattnig S, Guermazi A (2011) Advances in imaging of osteoarthritis and cartilage. Radiology 260:332-354

10. Hunter DJ, Guermazi A, Lo GH et al (2011) Evolution of semiquantitative whole joint assessment of knee OA: MOAKS (MRI Osteoarthritis Knee Score). Osteoarthritis Cartilage 19:990-1002

11. Oei EHG, van Tiel J, Robinson WH, Gold GE (2014) Quantitative radiologic imaging techniques for articular cartilage composition: toward early diagnosis and development of disease-modifying therapeutics for osteoarthritis. Arthritis Care Res 66:1129-1141

12. Pedoia V, Majumdar S (2019) Translation of morphological and functional musculoskeletal imaging. J Orthop Res 37:23-34

13. Roemer FW, Demehri S, Omoumi P et al (2020) State of the Art: Imaging of Osteoarthritis-Revisited 2020. Radiology 296:5-21

14. Starmans MPA, van der Voort SR, Tovar JMC, Veenland JF, Klein S, Niessen WJ (2020) Radiomics: data mining using quantitative medical image features. Handbook of Medical Image Computing and Computer Assisted Intervention. Elsevier, pp 429-456

15. MacKay JW, Kapoor G, Driban JB et al (2018) Association of subchondral bone texture on magnetic resonance imaging with radiographic knee osteoarthritis progression: data from the Osteoarthritis Initiative Bone Ancillary Study. Eur Radiol 28:4687-4695

16. MacKay JW, Murray PJ, Kasmai B, Johnson G, Donell ST, Toms AP (2016) MRI texture analysis of subchondral bone at the tibial plateau. Eur Radiol 26:3034-3045

17. MacKay JW, Murray PJ, Low SBL et al (2016) Quantitative analysis of tibial subchondral bone: texture analysis outperforms conventional trabecular microarchitecture analysis. J Magn Reson Imaging 43:1159-1170

18. Bowes MA, Kacena K, Alabas OA et al (2020) Machine-learning, MRI bone shape and important clinical outcomes in osteoarthritis: data from the Osteoarthritis Initiative. Ann Rheum Dis. https://doi. org/10.1136/annrheumdis-2020-217160

19. MacKay JW, Murray PJ, Kasmai B, Johnson G, Donell ST, Toms AP (2017) Subchondral bone in osteoarthritis: association between MRI texture analysis and histomorphometry. Osteoarthritis Cartilage 25: 700-707

20. Schiphof D, van Middelkoop M, de Klerk BM et al (2014) Crepitus is a first indication of patellofemoral osteoarthritis (and not of tibiofemoral osteoarthritis). Osteoarthritis Cartilage 22:631-638

21. Schiphof D, Oei EHG, Hofman A, Waarsing JH, Weinans H, BiermaZeinstra SMA (2014) Sensitivity and associations with pain and body weight of an MRI definition of knee osteoarthritis compared with radiographic Kellgren and Lawrence criteria: a population-based study in middle-aged females. Osteoarthritis Cartilage 22:440-446

22. Ikram MA, Brusselle GGO, Murad SD et al (2017) The Rotterdam Study: 2018 update on objectives, design and main results. Eur J Epidemiol 32:807-850

23. Runhaar J, Schiphof D, van Meer B, Reijman M, Bierma-Zeinstra SMA, Oei EHG (2014) How to define subregional osteoarthritis progression using semi-quantitative MRI Osteoarthritis Knee Score (MOAKS). Osteoarthritis Cartilage 22:1533-1536

24. Hunter DJ, Arden N, Conaghan PG et al (2011) Definition of osteoarthritis on MRI: results of a Delphi exercise. Osteoarthritis Cartilage 19:963-969

25. Emery CA, Whittaker JL, Mahmoudian A et al (2019) Establishing outcome measures in early knee osteoarthritis. Nat Rev Rheumatol $15: 438-448$

26. Xu D, van der Voet J, Hansson M et al (2020) Association between meniscal volume and development of knee osteoarthritis.
Rheumatology (Oxford). https://doi.org/10.1093/rheumatology/ keaa522

27. Van der Lijn F, De Bruijne M, Klein S et al (2011) Automated brain structure segmentation based on atlas registration and appearance models. IEEE Trans Med Imaging 31:276-286

28. Klein S, Staring M, Murphy K, Viergever MA, Pluim JPW (2009) Elastix: a toolbox for intensity-based medical image registration. IEEE Trans Med Imaging 29:196-205

29. Burr DB (2004) Anatomy and physiology of the mineralized tissues: role in the pathogenesis of osteoarthrosis. Osteoarthritis Cartilage 12: 20-30

30. Madry H, van Dijk CN, Mueller-Gerbl M (2010) The basic science of the subchondral bone. Knee Surg Sports Traumatol Arthrosc 18: 419-433

31. Starmans MPA, Miclea RL, van der Voort SR, Niessen WJ, Thomeer MG, Klein S (2018) Classification of malignant and benign liver tumors using a radiomics approach. Medical Imaging 2018: Image Processing. International Society for Optics and Photonics, p 105741D

32. Zou KH, Warfield SK, Bharatha A et al (2004) Statistical validation of image segmentation quality based on a spatial overlap index1: scientific reports. Acad Radiol 11:178-189

33. Kerkhof HJM, Bierma-Zeinstra SMA, Arden NK et al (2014) Prediction model for knee osteoarthritis incidence, including clinical, genetic and biochemical risk factors. Ann Rheum Dis 73:2116-2121

34. Friedman J, Hastie T, Tibshirani R (2010) Regularization paths for generalized linear models via coordinate descent. J Stat Softw 33:1-22

35. Zou H, Hastie T (2005) Regularization and variable selection via the elastic net. J R Stat Soc Series B Stat Methodology 67:301-320

36. Nadeau C, Bengio Y (2000) Inference for the generalization error. Adv Neural Inf Process Syst, pp 307-313

37. Kuhn M (2008) Building predictive models in $\mathrm{R}$ using the caret package. J Stat Softw 28:1-26

38. Grau J, Grosse I, Keilwagen J (2015) PRROC: computing and visualizing precision-recall and receiver operating characteristic curves in R. Bioinformatics 31:2595-2597

39. Saito T, Rehmsmeier M (2017) Precrec: fast and accurate precision-recall and ROC curve calculations in R. Bioinformatics $33: 145-147$

40. McAlindon TE, Snow S, Cooper C, Dieppe PA (1992) Radiographic patterns of osteoarthritis of the knee joint in the community: the importance of the patellofemoral joint. Ann Rheum Dis 51:844-849

41. Wise BL, Niu J, Yang M et al (2012) Patterns of compartment involvement in tibiofemoral osteoarthritis in men and women and in whites and African Americans. Arthritis Care Res 64:847-852

42. Kraus VB, Feng S, Wang $\mathrm{S}$ et al (2013) Subchondral bone trabecular integrity predicts and changes concurrently with radiographic and magnetic resonance imaging-determined knee osteoarthritis progression. Arthritis Rheum 65:1812-1821

43. Kraus VB, Collins JE, Charles HC et al (2018) Predictive validity of radiographic trabecular bone texture in knee osteoarthritis: the Osteoarthritis Research Society International/Foundation for the National Institutes of Health Osteoarthritis Biomarkers Consortium. Arthritis Rheumatol 70:80-87

44. Janvier T, Jennane R, Toumi H, Lespessailles E (2017) Subchondral tibial bone texture predicts the incidence of radiographic knee osteoarthritis: data from the osteoarthritis initiative. Osteoarthritis Cartilage 25:2047-2054

45. Hirvasniemi J, Niinimäki J, Thevenot J, Saarakkala S (2019) Bone density and texture from minimally post-processed knee radiographs in subjects with knee osteoarthritis. Ann Biomed Eng 47: $1181-1190$

46. Thomson J, O'Neill T, Felson D, Cootes T (2015) Automated shape and texture analysis for detection of osteoarthritis from radiographs 
of the knee. International Conference on Medical Image Computing and Computer-Assisted Intervention. Springer, pp 127-134

47. Peura M, Iivarinen J (1997) Efficiency of simple shape descriptors. Aspects of visual form 443-451

48. Grigorescu SE, Petkov N, Kruizinga P (2002) Comparison of texture features based on Gabor filters. IEEE Trans Image Process 11: $1160-1167$

49. Lu RS, Dennison E, Denison H, Cooper C, Taylor M, Bottema MJ (2018) Texture analysis based on Gabor filters improves the estimate of bone fracture risk from DXA images. Comput Methods Biomech Biomed Eng Imaging Vis 6:453-464

50. Ambellan F, Tack A, Ehlke M, Zachow S (2019) Automated segmentation of knee bone and cartilage combining statistical shape knowledge and convolutional neural networks: Data from the Osteoarthritis Initiative. Med Image Anal 52:109-118

Publisher's note Springer Nature remains neutral with regard to jurisdictional claims in published maps and institutional affiliations. 